\title{
Heritability of social anxiety disorder: a systematic review of methodological designs
}

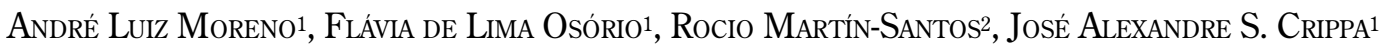 \\ 1 Graduate Program in Mental Health, Ribeirão Preto Medical School, University of São Paulo (USP), São Paulo, SP, Brazil. \\ 2 Service of Psychiatry and Psychology, Hospital Clinic, Institut d'Investigacions Biomèdiques August Pi i Sunver (Idibaps), Centro de Investigación en Red de Salud Mental (Cibersam), and \\ Department of Psychiatry and Clinical Psychobiology, University of Barcelona.
}

Study developed in Graduate Program in Mental Health, Ribeirão Preto Medical School, University of São Paulo.

Received: 8/6/2016 - Accepted: 5/9/2016

DOI: 10.1590/0101-60830000000090

\begin{abstract}
Background: The investigation of heritability stands out as an important means to establish the weight of genetic and environmental factors in the development of social anxiety disorder. Objective: This study aims to make a critical review of methodological designs used in the investigation of the social anxiety disorder (SAD) heritability. Methods: We reviewed 31 research articles published until October 2015 and found through the electronic search bases PubMed, Web of Science, and Scopus and manual searches in the reference lists of the selected references. Most of the investigations involved adult samples and twins to assess heritability. Results: There was great variability in the screening and diagnostic instruments used in the studies, leading to different outcomes. Structural equation models proved to be the most adequate to assess SAD heritability, allowing better estimates of this aspect of the disorder. SAD heritability rates varied between $13 \%$ and $76 \%$ in the articles reviewed. Discussion: We discuss methodological aspects that may affect the quality and the development of improved studies to investigate SAD heritability such as sample size, quality of screening instruments, and use of diagnostic interviews. More homogeneous investigations involving larger samples and standardized instruments and methods are desirable and opportune.
\end{abstract}

Moreno AL et al. / Arch Clin Psychiatry. 2016;43(4):83-92

Keywords: Social anxiety disorder, heritability, genetics, methodological design, social phobia.

\section{Introduction}

Social anxiety disorder (SAD) or social phobia is an anxiety disorder characterized by the presence of fear and anxiety in social situations, associated with the avoidance of such situations or significant personal distress that affect daily life. The prevalence of SAD has been estimated between $7 \%-12 \%^{1,2}$, causing impairment in the life of diagnosed individuals ${ }^{3}$ and direct and indirect costs to health systems ${ }^{4}$.

The current model of the development and maintenance of SAD holds that multiple factors interact at specific moments and circumstances for the disorder to occur ${ }^{5}$. This interaction model combines both genetic factors, such as polymorphisms ${ }^{6}$ and temperament ${ }^{7}$, and environmental factors including the perception of family environment ${ }^{8}$ and parental conflicts 9 . However, despite the existence of studies in this field, it is not yet possible to determine the specific contribution of each factor for the development of SAD. Therefore, the investigation of heritability stands out as an important means to establish the weight of genetic and environmental factors in the development of disorders, and different methodological designs can be used for this purpose.

In one methodological approach, heritability can be assessed by the chance that one relative will have the disorder, given the diagnosis of a patient, compared to a volunteer without the disorder (odds ratio). In this case, the participation of several relatives is required for each participant, divided according to the degree of genetic similarity (e.g.: 50\% genetic similarity for first-degree relatives, $25 \%$ for seconddegree relatives, 12.5 for third-degree relatives, and zero for spouses and adopted siblings). In this study design, the comparison of odds ratios (relative risk) for each degree of genetic similarity indicates the importance of genetic factors, whereas the analysis of relatives with no genetic similarity allows the observation of environmental factors. However, this design makes it difficult to determine the specific weight of genetic and environmental factors, since it is impossible to establish which environmental factors are social/shared or individual, as well as whether any genetic factors associated with the disorder are related to additive effects or dominant alleles.

Another method to assess heritability involves the participation of twins. In these studies, the genetic similarity between monozygotic (MZ) and dizygotic (DZ) twins is used to determine shared elements, since MZ twins virtually share $100 \%$ of their genes, while $\mathrm{DZ}$ twins share around half of their genes. It is also possible to explore gender differences in this type of investigation given the different genderrelated experiences of the twins. Studies involving twins allow a more accurate determination of the factors that influence a given disorder, which are observed according to four possible outcomes: additive genetic effects, genetic effects resulting from dominant or non-additive alleles, familial or shared environmental effects (e.g.: parental conflict), and environmental effects that are not shared by individuals (e.g.: perception of family environment).

In research with twins, heritability can be assessed through types of analyses. The first involves the correlations of a given disorder indicator (diagnosis, interview, instrument) between $\mathrm{MZ}$ and $\mathrm{DZ}$ twins. In this approach, differences indicate additive genetic effects (A), which can be considered dominant (D) if the correlation in MZ twins is at least twice as that of DZ twins. In another type of analysis, the factors are explored in structural equation models that indicate the model that best fits the sample data (e.g.: a model considering only additive genetic effects and environmental factors that are not shared; or another model that will also contemplate shared environmental factors) and the estimated explained percentage for each factor.

In respect to $\mathrm{SAD}$, a meta-analysis ${ }^{10}$ estimated the heritability of the disorder as ranging between $20 \%-40 \%$. This result, however, refers to a group comprising individuals with $\mathrm{SAD}$, specific phobia, and agoraphobia, and does not specify the heritability of each disorder. A more recent review estimated the heritability of SAD between $27 \%$ $56 \%{ }^{11}$, but it included only studies with twins and which described precise estimates of variance explained by genetic factors.

Despite these efforts and as far as we know, no study to date made a comprehensive assessment of investigations on the heritability

Address for correspondence: José Alexandre S. Crippa. Hospital das Clínicas da Faculdade de Medicina de Ribeirão Preto da Universidade de São Paulo - Campus Universitário, s/n, Monte Alegre

- 14048-900 - Ribeirão Preto, SP, Brazil. E-mail: moreno.andreluiz@gmail.com 
of SAD that used different methodological designs. Therefore, the objective of our study was to review articles dealing with the heritability of SAD with an emphasis on their methodological design and to provide directions for future research.

\section{Method}

Systematic searches were performed using the online databases PubMed, Web of Science, and Scopus for articles published until October 2015 using the following search terms: "social phobia AND genetics", "social phobia AND heritability", "social anxiety AND genetics", and "social anxiety AND heritability". The reference lists of articles found through the electronic databases and other review articles were hand-searched for additional references.

We included articles that described any measure or SAD heritability as an outcome. Animal studies, studies on genetic polymorphisms related to $\mathrm{SAD}$, articles with no specific SAD heritability data, letters to the editor, editorials, book chapters, and review articles were not included in this review.

From each selected article, the following data were extracted whenever possible: (I) origin of the study sample; (II) country; (III) sample size; (IV) instruments used for the screening or diagnosis of SAD; (V) method of data analysis; (VI) primary outcome; (VII) correlation values between $\mathrm{MZ}$ and $\mathrm{DZ}$ twins; (VIII) genetic model; and (IX) values of additive genetic effects (A), shared environmental effects (C), and environmental effects (E). The data of the different articles were than assessed conjointly based on these categories. In addition, the studies were assessed according to the criteria of the STROBE initiative ${ }^{12}$, which consist of a list of 22 items that assess the methodological quality of scientific articles.

\section{Results}

A total of 888 articles were found through the electronic searches. After a selection based on the inclusion and exclusion criteria, 28 articles were included in the review. Three other articles were found through the reference lists of selected articles and were also included in the review. Therefore, this review comprises the data of 31 articles. Details of the search and selection procedures are presented in Figure 1.

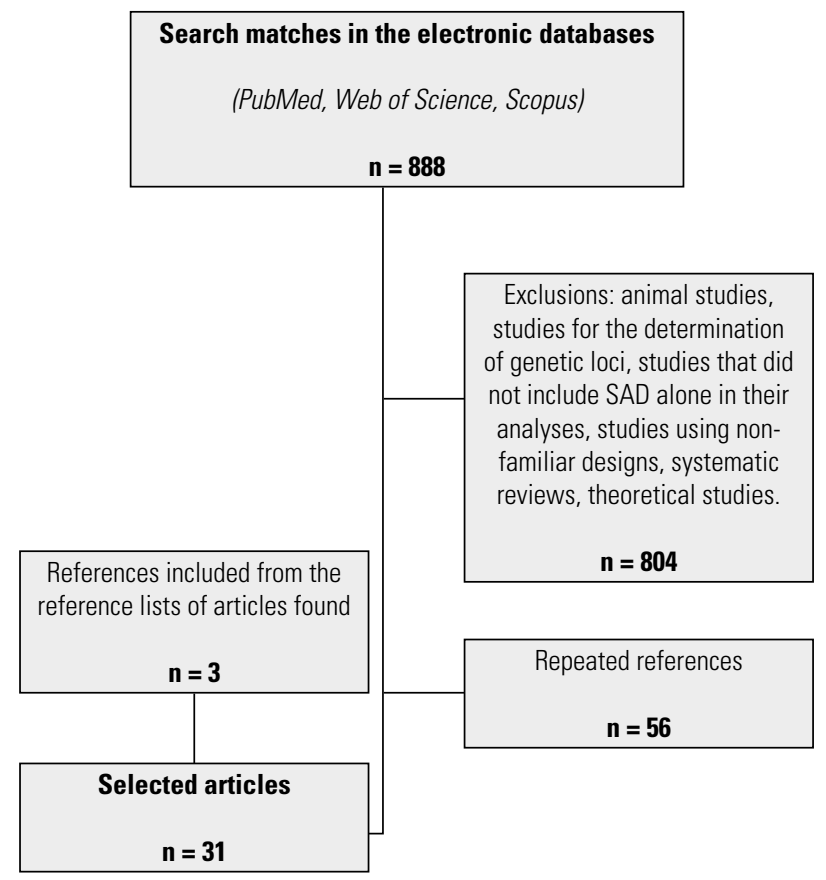

Figure 1. Flowchart showing the steps of the search and selection of articles for the review.
Table 1 presents the data of each article included in the review. From the studies selected, 21 (67.75\%) recruited twins, and only one of these included volunteers recruited through community ads. Thus, 20 (95.23\%) of the studies involving twins used public or private medical records, where data on the twin pairs were available since their birth until the data collection. In respect to the origin of articles included in this review, 19 (61.3\%) were from Europe, 9 (29.03\%) were from North America, and 2 (6.4\%) came from Oceania. One study involved samples from two continents (Europe and Oceania).

Concerning the age range of participants, 17 studies (54.83\%) involved adult samples and 12 studies (38.7\%) involved children and adolescents. Two longitudinal studies collected data from participants at different age ranges, including periods of childhood, adolescence, and adult life. In general, the instruments used to measure symptoms or diagnose SAD varied widely across the studies. Despite this variation, self-report instruments were used as the only measure of SAD symptoms in 13 studies (41.93\%). Thus, whereas some of the studies reviewed used well-established measures for the assessment of SAD (e.g., Social Phobia Inventory $\left.{ }^{13}\right)$, others used sets of items taken from assessment instruments (e.g. $\left.{ }^{14}\right)$. Interviews were used as the only diagnostic instrument in 11 studies $(35.48 \%)$ and structured interviews as the Structured Clinical Interview for DSM-IV (SCID) ${ }^{15}$ and the Composite International Diagnostic Interview (CIDI) ${ }^{16}$ were the most common among these. Two investigations (6.4\%) used only medical records to establish diagnosis, with no procedures included to confirm the criteria applied or the accuracy of diagnosis during the period of the study. Other studies used more than one type of instrument to assess symptoms or diagnosis due to different profiles of their participants. In these studies, cases and controls were assessed with diagnostic interviews (three studies) or self-report instruments (two studies), whereas relatives (parents, siblings, uncles and grandparents) were assessed through previous diagnostic records or assessments by their health networks.

The studies included in this review also differed significantly in respect to methods of data analysis and the outcomes derived from these analyses. Among all the articles selected, 14 (45.16\%) analyzed correlations between the twins in their samples, using the correlations between monozygotic and dizygotic siblings as outcomes, and also provided models and heritability estimates as outcomes based on structural equations. Two studies assessed only correlation differences between twins, while five others reported only heritability estimates based on structural equation models. Five of the articles reviewed analyzed the odds ratios for SAD in relatives of participants diagnosed with the disorder and presented as outcomes the differences between the degrees of relatedness, including that of twins, considering cases and controls. In addition, two studies reported heritability estimates obtained from structural equation models together with odds ratios for relatives of affected patients, and only one study provided heritability estimates based on the difference of correlations between siblings and half-siblings together with odds ratios. The investigation by Stein et al. ${ }^{17}$ used factorial analysis to assess the extent to which the degree of consanguinity of SAD patients explained SAD symptoms in relatives, presenting as their outcome the variance explained by consanguinity relative to other factors. Finally, the study by Li et al. ${ }^{18}$ assessed the incidence of SAD in siblings of patients diagnosed with anxiety disorders, presenting the standardized index ratio as outcome.

All the articles included in this review had satisfactory methodological quality, fulfilling at least $15(68 \%)$ of the STROBE assessment items ${ }^{12}$. Additionally, 17 articles (55\%) fulfilled more than $80 \%$ of the methodological recommendations of that initiative. Conversely, none of the studies included followed all the guidelines of the STROBE. The mains items that were not fulfilled by the studies reviewed here refer to the absence of information on sample size calculations and efforts to reduce possible biases. 
Table 1. Population, number of participants, age of participants, instruments used to assess SAD, data analysis method, outcome and items fulfilled of STROBE of each study included

\begin{tabular}{|c|c|c|c|c|c|c|c|}
\hline Reference & Country/Population & Participants & $\begin{array}{c}\text { Age } \\
(\text { Mean } \pm S D)\end{array}$ & Instruments & Data analysis & Outcome & $\begin{array}{l}\text { STROBE items } \\
\text { fulfilled } n(\%)\end{array}$ \\
\hline $\begin{array}{l}\text { Coelho et al., } \\
200721\end{array}$ & UK/Outpatients & $\begin{array}{c}37 \mathrm{SAD} \\
22 \mathrm{GAD} \\
15 \mathrm{SAD}+\mathrm{GAD} \\
60 \mathrm{HC} \\
403 \text { Relatives }\end{array}$ & $\begin{array}{c}\text { Cases: } 32.1 \\
\text { (3.77) } \\
\text { Relatives: } 47.4 \\
\text { (14.98) }\end{array}$ & $\begin{array}{c}\text { SCID. Diagnosis } \\
\text { of relatives based } \\
\text { on information } \\
\text { provided by cases/ } \\
\text { controls }\end{array}$ & OR Case $X$ Relative & $\begin{array}{c}\text { OR SAD X SAD = } \\
\text { 3.38 (1.25-9.16); } \\
\text { SAD X Comorbidity } \\
\text { OR (SAD-GAD) = } \\
\text { 3.50 (0.98-12.55); } \\
\text { Comorbidity OR } \\
\text { (SAD-GAD) X TAS } \\
=7.01 \text { (0.82-60.23); } \\
\text { Comorbidity OR } \\
\text { X Comorbidity = } \\
\text { 17.34 (1.96-153.62) }\end{array}$ & $19(86 \%)$ \\
\hline $\begin{array}{l}\text { Czajkowski et al., } \\
201122\end{array}$ & $\begin{array}{c}\text { Norway/Twins } \\
\text { (medical record } \\
\text { services) }\end{array}$ & $\begin{array}{l}446 \mathrm{P} \mathrm{Mz} F ; \\
264 \mathrm{P} \mathrm{Dz} F ; \\
10 \mathrm{I}\end{array}$ & 28.1 & CIDI & \begin{tabular}{|c|} 
Correlations \\
between $\mathrm{Mz}$ \\
and $\mathrm{Dz}$ \\
Structural Equation \\
Models
\end{tabular} & $\begin{array}{c}\text { Correlation } \\
\text { differences } \\
\text { Heritability } \\
\text { estimates }\end{array}$ & $20(91 \%)$ \\
\hline Eley et al., 200323 & $\begin{array}{l}\text { UK/Twins (medical } \\
\text { record services) }\end{array}$ & $\begin{array}{l}723 \text { P Mz M; } \\
769 \text { P Dz M; } \\
818 \text { P Mz F; } \\
760 \text { P Dz F; } \\
1494 \text { P Dz } 0 \\
\end{array}$ & 4 & $\begin{array}{c}16 \text {-item } \\
\text { questionnaire on } \\
\text { anxiety-related } \\
\text { behaviors }\end{array}$ & \begin{tabular}{|c|} 
Correlations \\
between $\mathrm{Mz}$ \\
and $\mathrm{Dz}$ \\
Structural Equation \\
Models \\
\end{tabular} & $\begin{array}{c}\text { Correlation } \\
\text { differences } \\
\text { Heritability } \\
\text { estimates }\end{array}$ & $18(82 \%)$ \\
\hline Eley et al., $2008^{24}$ & $\begin{array}{l}\text { UK/Twins (medical } \\
\text { record services) }\end{array}$ & $\begin{array}{c}\text { T0: } \\
754 \text { P Mz M; } \\
783 \text { P Dz M; } \\
\text { 845 P Mz F; } 768 \text { P } \\
\text { Dz F; 1512 P Dz 0; } \\
\text { T1: } \\
\text { 120 P Mz M; } \\
133 \text { P Mz F; } \\
138 \text { P Dz M; } \\
\text { 136 P Dz F; } \\
\text { 327 P Dz 0 }\end{array}$ & $\begin{array}{l}\text { T0: } 4 \\
\text { T1: } 6\end{array}$ & $\begin{array}{c}\text { T0 } \\
\text { Anxiety Related } \\
\text { Behaviors } \\
\text { Questionnaire. } \\
\text { T1 } \\
\text { Anxiety Disorders } \\
\text { Interview Schedule } \\
\text { for Children and } \\
\text { Parents }\end{array}$ & \begin{tabular}{|c|} 
Correlations \\
between $\mathrm{Mz}$ \\
and $\mathrm{Dz}$ \\
Structural Equation \\
Models
\end{tabular} & $\begin{array}{c}\text { Correlation } \\
\text { differences } \\
\text { Heritability } \\
\text { estimates }\end{array}$ & $18(82 \%)$ \\
\hline $\begin{array}{l}\text { Hallett et al., } \\
2009^{14}\end{array}$ & $\begin{array}{l}\text { UK/Twins (medical } \\
\text { record services) }\end{array}$ & $\begin{array}{c}\text { T0 } \\
1205 \text { P Mz M; } \\
1118 \text { P Dz M; } \\
1370 \text { P Mz F; } \\
1219 \text { P Dz F; } \\
2255 \text { P Dz 0; } \\
\text { T1: } \\
538 \text { P Mz M; } \\
674 \text { P Dz M; } \\
503 \text { P Mz F; } \\
557 \text { P Dz F; } \\
1004 \text { P Dz } 0\end{array}$ & $\begin{array}{l}\text { T0: } 7 \\
\text { T1: } 9\end{array}$ & $\begin{array}{l}25 \text { items taken } \\
\text { from other } \\
\text { instruments }\end{array}$ & \begin{tabular}{|c|} 
Correlations \\
between $\mathrm{Mz}$ \\
and $\mathrm{Dz}$ \\
Structural Equation \\
Models
\end{tabular} & $\begin{array}{c}\text { Correlation } \\
\text { differences } \\
\text { Heritability } \\
\text { estimates }\end{array}$ & $20(91 \%)$ \\
\hline $\begin{array}{l}\text { Hallett et al., } \\
201225\end{array}$ & $\begin{array}{l}\text { UK/Twins (medical } \\
\text { record services) }\end{array}$ & $\begin{array}{c}\text { T0: } \\
\text { 1232 P Mz M; } \\
\text { 1164 P Dz M; } \\
1375 \text { P Mz F; } \\
1230 \text { P Dz F; } \\
2310 \text { P DzO; } \\
\text { T1: } \\
\text { 1069P Mz M; } \\
\text { 1044 P Dz M; } \\
\text { 1195 P Mz F; } \\
\text { 1054 P Dz F; } \\
2064 \text { P Dz O }\end{array}$ & $\begin{array}{l}\text { T0: } 7 \\
\text { T1: } 9\end{array}$ & $\begin{array}{c}22 \text { items taken } \\
\text { from other } \\
\text { instruments }\end{array}$ & \begin{tabular}{|c|} 
Correlations \\
between $\mathrm{Mz}$ \\
and $\mathrm{Dz}$ \\
Structural Equation \\
Models
\end{tabular} & $\begin{array}{c}\text { Correlation } \\
\text { differences } \\
\text { Heritability } \\
\text { estimates }\end{array}$ & $18(82 \%)$ \\
\hline \begin{tabular}{|l|} 
Hettema et al., \\
$2005^{26}$
\end{tabular} & $\begin{array}{l}\text { USA/Community } \\
\text { sample }\end{array}$ & $\begin{array}{l}2156 \mathrm{PF} ; \\
2939 \mathrm{PM}\end{array}$ & - & $\begin{array}{c}\text { Diagnostic } \\
\text { interviews based } \\
\text { on DSM = III-R } \\
\text { criteria }\end{array}$ & $\begin{array}{l}\text { Structural Equation } \\
\text { Models }\end{array}$ & $\begin{array}{l}\text { Heritability } \\
\text { estimates }\end{array}$ & $17(77 \%)$ \\
\hline
\end{tabular}




\begin{tabular}{|c|c|c|c|c|c|c|c|}
\hline Reference & Country/Population & Participants & $\begin{array}{c}\text { Age } \\
(\text { Mean } \pm S D)\end{array}$ & Instruments & Data analysis & Outcome & $\begin{array}{l}\text { STROBE items } \\
\text { fulfilled } n(\%)\end{array}$ \\
\hline $\begin{array}{l}\text { Hettema et al., } \\
200627\end{array}$ & $\begin{array}{l}\text { USA/Twins } \\
\text { (medical record } \\
\text { services) }\end{array}$ & $\begin{array}{l}679 \text { P Mz F; } \\
467 \text { P Dz F; } \\
869 \text { P Mz M; } \\
653 \text { P Dz M; } \\
1429 \text { P Dz 0; } \\
125 \text { I Mz F; } \\
56 \text { I Dz F; } \\
230 \text { I Mz M; } \\
275 \text { I Dz M; } \\
462 \text { I Dz O }\end{array}$ & - & $\begin{array}{c}\text { Diagnostic } \\
\text { interviews based } \\
\text { on DSM = III-R } \\
\text { criteria }\end{array}$ & $\begin{array}{l}\text { Structural Equation } \\
\text { Models }\end{array}$ & $\begin{array}{l}\text { Heritability } \\
\text { estimates }\end{array}$ & $18(82 \%)$ \\
\hline $\begin{array}{l}\text { Hudson et al., } \\
2003^{28}\end{array}$ & $\begin{array}{c}\text { Austria/Outpatient } \\
\text { services }\end{array}$ & $\begin{array}{c}64 \mathrm{MDD} \\
58 \mathrm{HC} \\
152 \text { relatives }\end{array}$ & $\begin{array}{c}\text { Cases: } \\
39.5 \text { (15) } \\
\text { Relatives (cases): } \\
39.6 \text { (13.7) } \\
\text { Controls: } \\
40.9 \text { (14.1). } \\
\text { Relatives (controls): } \\
37.4 \text { (13.1) } \\
\end{array}$ & SCID & OR Case X Relative & $\begin{array}{c}\text { OR without } \\
\text { depression = } \\
4.6(1.2-18) ; 0 R \\
\text { with comorbid } \\
\text { depression = 2.7 } \\
(0.59-12)\end{array}$ & $17(77 \%)$ \\
\hline $\begin{array}{l}\text { Isomura et al., } \\
2015^{29}\end{array}$ & \begin{tabular}{|} 
Sweden/Population \\
records with \\
mental disorder \\
diagnoses
\end{tabular} & \begin{tabular}{|c|} 
18399 SAD \\
$2673 \mathrm{APD}$ \\
$210.720 \mathrm{HC}$ \\
2.959.278 Relatives \\
\end{tabular} & - & $\begin{array}{l}\text { Previous diagnoses } \\
\text { in medical records }\end{array}$ & $\begin{array}{l}\text { OR Case X Relative } \\
\text { Correlations } \\
\text { between siblings } \\
\text { and half-siblings }\end{array}$ & $\begin{array}{c}\text { OR First-degree = } \\
4.74 \text { (4.28-5.25). } \\
\text { OR Second-degree } \\
=2.3(2.01-2.63) \text {. } \\
\text { OR Third-degree = } \\
1.72 \text { (1.52-1.94). } \\
\text { OR Non-biological } \\
\text { parents = 4.01 } \\
\text { (3.26-4.95). } \\
\text { Correlation for } \\
\text { siblings = 0.27; } \\
\text { half-siblings } \\
\text { (mother's side) = } \\
0.13 . \text { Heritability } \\
\text { estimated by the } \\
\text { correlation = } 0.56\end{array}$ & $17(77 \%)$ \\
\hline $\begin{array}{l}\text { Kendler et al., } \\
2001^{30}\end{array}$ & $\begin{array}{l}\text { USA/Twins } \\
\text { (medical record } \\
\text { services) }\end{array}$ & $\begin{array}{l}707 \text { P Mz M; } \\
290 \text { P Dz M; } \\
254 \text { I Mz M; } \\
290 \text { I Dz M }\end{array}$ & $36.8(9.1)$ & $\begin{array}{c}\text { Diagnostic } \\
\text { Interview Scale } \\
\text { (DIS). Version III-A }\end{array}$ & $\begin{array}{l}\text { OR Case X Sibling } \\
\text { Structural Equation } \\
\text { Models }\end{array}$ & $\begin{array}{c}\text { Differences in OR. } \\
\mathrm{MZ}=2.3(0.92- \\
5.77) ; \mathrm{DZ}=1.73 \\
(0.50-6.07)\end{array}$ & $14(64 \%)$ \\
\hline $\begin{array}{l}\text { Kendler et al., } \\
2008^{31}\end{array}$ & $\begin{array}{l}\text { Sweden/Twins } \\
\text { (medical record } \\
\text { services) }\end{array}$ & $\begin{array}{l}242 \text { P Mz F; } \\
182 \text { P Dz F; } \\
240 \text { P Mz M; } \\
168 \text { P Dz M; } \\
390 \text { P Dz O }\end{array}$ & $\begin{array}{c}\text { T0: } \\
13-14 \\
\text { T1: } \\
16-17 \\
\text { T2 } \\
19-20 \\
\end{array}$ & $\begin{array}{l}\text { Items dealing with } \\
\text { fear of specific } \\
\text { situations and } \\
\text { objects }\end{array}$ & $\begin{array}{c}\text { Correlations } \\
\text { between } \mathrm{Mz} \\
\text { and } \mathrm{Dz} \\
\text { Structural Equation } \\
\text { Models }\end{array}$ & $\begin{array}{c}\text { Correlation } \\
\text { differences } \\
\text { Heritability } \\
\text { estimates }\end{array}$ & $17(77 \%)$ \\
\hline $\begin{array}{l}\text { Knappe et al., } \\
2009_{32}\end{array}$ & $\begin{array}{l}\text { Germany/Cohort } \\
\text { population for } \\
\text { the study of } \\
\text { psychopathology }\end{array}$ & $\begin{array}{l}\text { T0: } 1395 \text { I } \\
\text { T1: } 1228 \text { I } \\
\text { T2: } 1169 \text { I } \\
\text { T3 1,022 I }\end{array}$ & $\begin{array}{l}\text { T0: } 14-17 \\
\text { T1: } 16-19 \\
\text { T2: } 18-21 \\
\text { T3: } 24-27\end{array}$ & CIDI & OR Case X Relative & $\begin{array}{c}\text { Symptomatic: OR } \\
=1.3(0.76-2.23) ; \\
\text { Subthreshold: } 0 R \\
=1.44 \text { (0.75-2.78); } \\
\text { Diagnosis: } 0 R= \\
3.21(1.21-8.49)\end{array}$ & $18(82 \%)$ \\
\hline Lahey et al., 201133 & $\begin{array}{c}\text { USA/Twins } \\
\text { (medical record } \\
\text { services) }\end{array}$ & $1571 \mathrm{PMz} / \mathrm{Dz}$ & 6-17 & $\begin{array}{c}\text { Child and } \\
\text { Adolescent } \\
\text { Psychopathology } \\
\text { Scale }\end{array}$ & $\begin{array}{l}\text { Structural Equation } \\
\text { Models }\end{array}$ & $\begin{array}{l}\text { Heritability } \\
\text { estimates }\end{array}$ & $17(77 \%)$ \\
\hline Li et al., 201118 & $\begin{array}{l}\text { Sweden/Population } \\
\text { records with } \\
\text { mental disorder } \\
\text { diagnoses }\end{array}$ & $\begin{array}{c}42602 \mathrm{AD} ; \\
2093 \text { relatives }\end{array}$ & & $\begin{array}{l}\text { Previous diagnoses } \\
\text { in medical records }\end{array}$ & $\begin{array}{c}\text { Standardized } \\
\text { incidence ratios }\end{array}$ & $\begin{array}{c}\text { Men: } 4.49 \text { (1.88- } \\
\text { 10.07); Women: } \\
2.51 \text { (0.7-7.35); } \\
\text { Total: } 3.68 \text { (1.68- } \\
\text { 7.69) }\end{array}$ & $17(77 \%)$ \\
\hline $\begin{array}{l}\text { López-Solà et al., } \\
2014^{34}\end{array}$ & $\begin{array}{l}\text { Australia/Twins } \\
\text { (medical record } \\
\text { services) }\end{array}$ & $\begin{array}{l}204 \text { P Mz M; } \\
299 \text { P Mz F; } \\
111 \text { P Dz M; } \\
194 \text { P Dz F; } \\
125 \text { I Mz M; } \\
150 \text { I Mz F; } \\
132 \text { I Dz M; } \\
192 \text { I Dz F; }\end{array}$ & $\begin{array}{l}\text { MZ: } 34.5(7.8) \\
\text { DZ: } 33.9(8)\end{array}$ & SPIN & $\begin{array}{c}\text { Correlations } \\
\text { between } \mathrm{Mz} \\
\text { and } \mathrm{Dz} \\
\text { Structural Equation } \\
\text { Models }\end{array}$ & $\begin{array}{l}\text { Correlation } \\
\text { differences } \\
\text { Heritability } \\
\text { estimates }\end{array}$ & $20(91 \%)$ \\
\hline
\end{tabular}




\begin{tabular}{|c|c|c|c|c|c|c|c|}
\hline Reference & Country/Population & Participants & $\begin{array}{c}\text { Age } \\
(\text { Mean } \pm \text { SD) }\end{array}$ & Instruments & Data analysis & Outcome & $\begin{array}{l}\text { STROBE items } \\
\text { fulfilled } n(\%)\end{array}$ \\
\hline Low et al., $2008^{35}$ & $\begin{array}{c}\text { USA/Patients } \\
\text { recruited from } \\
\text { outpatient services } \\
\text { and the community. } \\
\text { Relatives } \\
\text { contacted. }\end{array}$ & $\begin{array}{c}26 \text { I SAD + PD } \\
40 \text { I PD } \\
46 \text { I SAD } \\
32 \text { I AD } \\
81 \text { I HC. } 1053 \\
\text { relatives }\end{array}$ & $\begin{array}{c}\text { SAD + PD: } \\
39 \text { (5.9) } \\
\text { PD: } 39.5(5.2) \\
\text { SP: } 40.8(6.3) \\
\text { AD: } 40.4 \text { (6.2) } \\
\text { Controls: } 41 \text { (6.3) }\end{array}$ & \begin{tabular}{|c|} 
Schedule for \\
Affective Disorders \\
and Schizophrenia. \\
Family History- \\
Research \\
Diagnostic Criteria \\
\end{tabular} & $\begin{array}{l}\text { OR Case X } \\
\text { Relatives }\end{array}$ & $\begin{array}{c}\text { OR SP-SP }=1.8 \\
(1.1-2.9)\end{array}$ & $17(77 \%)$ \\
\hline Low et al., $2008^{36}$ & $\begin{array}{c}\text { USA/Patients } \\
\text { recruited from } \\
\text { outpatient services } \\
\text { and the community. } \\
\text { Relatives } \\
\text { contacted. }\end{array}$ & $\begin{array}{c}76 \text { I SAD } \\
60 \text { I HC } \\
620 \text { relatives }\end{array}$ & $\begin{array}{c}\text { Cases: } 39.9(5.3) \\
\text { Controls: } 40.9 \\
(6.28)\end{array}$ & $\begin{array}{l}\text { SCID for cases } \\
\text { and controls. } \\
\text { Best estimate } \\
\text { Diagnoses for } \\
\text { relatives }\end{array}$ & $\begin{array}{l}\text { OR Case X } \\
\text { Relatives }\end{array}$ & $\begin{array}{c}\text { Clinical OR = } \\
2.74(1.1-6.84) ; \\
\text { Community OR= } \\
2.38(0.91-6.22)\end{array}$ & $16(73 \%)$ \\
\hline $\begin{array}{l}\text { Michelini et al., } \\
2015^{37}\end{array}$ & $\begin{array}{l}\text { UK/Twins (medical } \\
\text { record services) }\end{array}$ & $\begin{array}{l}88 \text { P Mz M; } \\
134 \text { P Mz F; } \\
64 \text { P Dz M; } \\
130 \text { P Dz F; } \\
214 \text { P Dz 0; } \\
30 \text { P S M; } \\
51 \text { P S F; } \\
\text { 71 P S 0 } \\
\end{array}$ & $17(1.66)$ & $\begin{array}{l}\text { Spence Children's } \\
\text { Anxiety Scale }\end{array}$ & $\begin{array}{c}\text { Correlations } \\
\text { between } \mathrm{Mz} \\
\text { and } \mathrm{Dz} \\
\text { Structural Equation } \\
\text { Models }\end{array}$ & $\begin{array}{c}\text { Correlation } \\
\text { differences } \\
\text { Heritability } \\
\text { estimates }\end{array}$ & $21(95 \%)$ \\
\hline $\begin{array}{l}\text { Middedorp et al., } \\
2005^{38}\end{array}$ & $\begin{array}{l}\text { The Netherlands } \\
\text { and Australia/ } \\
\text { Twins (medical } \\
\text { record services) }\end{array}$ & $\begin{array}{l}1334 \text { I M; } \\
2088 \text { I F }\end{array}$ & $\begin{array}{c}\text { Men: } 35.15 \\
\text { Women: } 35.15\end{array}$ & CIDI & $\begin{array}{l}\text { Correlations } \\
\text { between } \mathrm{Mz} \\
\text { and } \mathrm{Dz}\end{array}$ & $\begin{array}{c}\text { Correlation } \\
\text { differences } \\
\text { Brothers: } 0.20 \\
\text { (0.09-0.31) } \\
\text { Sisters: } 0.20 \\
\text { (0.09-0.31) } \\
\text { Different gender: } \\
0.20 \text { (0.09-0.31) } \\
\end{array}$ & $20(91 \%)$ \\
\hline $\begin{array}{l}\text { Mosing et al., } \\
2009_{39}\end{array}$ & $\begin{array}{c}\text { Australia/Twins } \\
\text { (medical record } \\
\text { services) }\end{array}$ & $\begin{array}{l}1337 \mathrm{P} \mathrm{Mz} \\
1384 \mathrm{P} \mathrm{Dz}\end{array}$ & $\begin{array}{c}\text { Mz: } 44.07(12.4) ; \\
\text { Dz: } 29.9 \\
\text { (2.5) }\end{array}$ & \begin{tabular}{|c|} 
Computer \\
algorithms based \\
on responses in the \\
Semi-Structured \\
Assessment for \\
the Genetics of \\
Alcoholism (SSA- \\
GA) \\
\end{tabular} & $\begin{array}{l}\text { OR Case X Sibling } \\
\text { Structural Equation } \\
\text { Models }\end{array}$ & $\begin{array}{c}\text { Differences in OR. } \\
\mathrm{MZ}=11.9(3.7- \\
38.8) ; \mathrm{DZ}=1.5(0.2- \\
\text { 11.0). Heritability } \\
\text { estimates }\end{array}$ & $17(77 \%)$ \\
\hline $\begin{array}{l}\text { Nelson et al., } \\
2000^{40}\end{array}$ & $\begin{array}{c}\text { USA/Twins } \\
\text { (medical record } \\
\text { services) }\end{array}$ & 672 P F Mz/Dz & 18.2 & \begin{tabular}{|c|} 
Telephone \\
interview with \\
questions adapted \\
from the Diagnostic \\
Interview for \\
Children and \\
Adolescents \\
\end{tabular} & $\begin{array}{l}\text { Correlations } \\
\text { between } \mathrm{Mz} \\
\text { and } \mathrm{Dz}\end{array}$ & $\begin{array}{l}\text { Heritability } \\
\text { estimates }\end{array}$ & $18(82 \%)$ \\
\hline $\begin{array}{l}\text { Ogliari et al., } \\
2006^{41}\end{array}$ & $\begin{array}{c}\text { Italy/Twins } \\
\text { (medical record } \\
\text { services) }\end{array}$ & $\begin{array}{l}70 \text { P Mz M; } \\
65 \text { P Mz F; } \\
50 \text { P Dz M; } \\
78 \text { P Dz F; } \\
115 \text { P Dz } 0\end{array}$ & $13.03(2.6)$ & SCARED & $\begin{array}{c}\text { Correlations } \\
\text { between } \mathrm{Mz} \\
\text { and } \mathrm{Dz} \\
\text { Structural Equation } \\
\text { Models } \\
\end{array}$ & $\begin{array}{c}\text { Correlation } \\
\text { differences } \\
\text { Heritability } \\
\text { estimates }\end{array}$ & $20(91 \%)$ \\
\hline $\begin{array}{l}\text { Ogliari et al., } \\
2010^{42}\end{array}$ & $\begin{array}{c}\text { Italy/Twins } \\
\text { (medical record } \\
\text { services) }\end{array}$ & $\begin{array}{l}70 \text { P Mz M; } \\
65 \text { P Mz F; } \\
50 \text { P Dz M; } \\
78 \text { P Dz F; } \\
115 \text { P Dz } 0\end{array}$ & 8-17 & SCARED & $\begin{array}{c}\text { Correlations } \\
\text { between } \mathrm{Mz} \\
\text { and } \mathrm{Dz} \\
\text { Structural Equation } \\
\text { Models }\end{array}$ & $\begin{array}{c}\text { Correlation } \\
\text { differences } \\
\text { Heritability } \\
\text { estimates }\end{array}$ & $20(91 \%)$ \\
\hline $\begin{array}{l}\text { Reichborn- } \\
\text { Kjennerud et al., } \\
2007^{43}\end{array}$ & $\begin{array}{l}\text { Norway/Twins } \\
\text { (medical record } \\
\text { services) }\end{array}$ & $\begin{array}{l}898 \mathrm{P} \mathrm{Mz} F ; \\
529 \mathrm{P} \mathrm{Dz} F\end{array}$ & - & CIDI & $\begin{array}{c}\text { Correlations } \\
\text { between } \mathrm{Mz} \\
\text { and } \mathrm{Dz} \\
\text { Structural Equation } \\
\text { Models }\end{array}$ & $\begin{array}{c}\text { Correlation } \\
\text { differences } \\
\text { Heritability } \\
\text { estimates }\end{array}$ & $20(91 \%)$ \\
\hline Skre et al., 200044 & $\begin{array}{l}\text { Norway/Twins } \\
\text { (medical record } \\
\text { services) }\end{array}$ & $\begin{array}{l}17 \mathrm{P} \mathrm{Mz} F ; \\
6 \text { I Mz M; } \\
21 \text { I Dz F; } \\
17 \mathrm{Izz} \mathrm{M}\end{array}$ & $\begin{array}{l}41 \\
(9)\end{array}$ & $\begin{array}{l}\text { Items dealing with } \\
\text { fear of specific } \\
\text { situations and } \\
\text { objects }\end{array}$ & $\begin{array}{c}\text { Correlations } \\
\text { between } \mathrm{Mz} \\
\text { and } \mathrm{Dz} \\
\text { Structural Equation } \\
\text { Models }\end{array}$ & $\begin{array}{c}\text { Correlation } \\
\text { differences } \\
\text { Heritability } \\
\text { estimates }\end{array}$ & $20(91 \%)$ \\
\hline
\end{tabular}




\begin{tabular}{|c|c|c|c|c|c|c|c|}
\hline Reference & Country/Population & Participants & $\begin{array}{c}\text { Age } \\
(\text { Mean } \pm S D)\end{array}$ & Instruments & Data analysis & Outcome & $\begin{array}{l}\text { STROBE items } \\
\text { fulfilled } n(\%)\end{array}$ \\
\hline Stein et al., 200117 & $\begin{array}{l}\text { Canada/Patients } \\
\text { recruited from } \\
\text { outpatient services } \\
\text { and the community. } \\
\text { Relatives contacted }\end{array}$ & $\begin{array}{c}31 \text { I SAD } \\
24 \text { I HC } \\
65 \text { relatives }\end{array}$ & $\begin{array}{c}\text { Cases: } 42.5 \text { (16.8) } \\
\text { Controls: } 40.7 \\
\text { (15.6) }\end{array}$ & $\begin{array}{l}\text { SCID (cases); } \\
\text { Fear of Negative } \\
\text { Evaluation Scale; } \\
\text { Social Phobia } \\
\text { Scale; Social } \\
\text { Interactional } \\
\text { Anxiety Scale } \\
\text { (subjects) } \\
\text { Scale, Social } \\
\text { Interactional } \\
\text { Anxiety Scale }\end{array}$ & Factorial analysis & $\begin{array}{l}\text { Being a first- } \\
\text { degree relative } \\
\text { explains } 84 \% \text { of } \\
\text { the variance }\end{array}$ & $15(68 \%)$ \\
\hline Stein et al., 200245 & $\begin{array}{l}\text { Canada/Twins } \\
\text { (community } \\
\text { advertisement) }\end{array}$ & $\begin{array}{c}55 \text { P Mz M; } \\
154 \text { P Mz F; } \\
30 \text { P Dz M; 115P } \\
\text { Dz F; } \\
\text { 35 P Dz } 0\end{array}$ & $\begin{array}{c}\text { MzM: } 36.66 \text { (16.86) } \\
\text { MzF: } 34.82 \text { (14.1) } \\
\text { DzM: } 31.53 \text { (13.37) } \\
\text { DzF: } \\
32.38 \text { (13.13) Dz0: } \\
29.88 \text { (12.82) }\end{array}$ & $\begin{array}{l}\text { Brief Fear } \\
\text { of Negative } \\
\text { Evaluation }\end{array}$ & $\begin{array}{c}\text { Correlations } \\
\text { between } \mathrm{Mz} \\
\text { and } \mathrm{Dz} \\
\text { Structural Equation } \\
\text { Models }\end{array}$ & $\begin{array}{c}\text { Correlation } \\
\text { between twins } \\
\text { Heritability } \\
\text { estimates }\end{array}$ & $17(77 \%)$ \\
\hline $\begin{array}{l}\text { Trzaskowski et al., } \\
2012^{46}\end{array}$ & $\begin{array}{l}\text { UK/Twins (medical } \\
\text { record services) }\end{array}$ & $\begin{array}{l}\text { T0: } 7834 \text { I } \\
\text { T1: } 3644 \text { I }\end{array}$ & $\begin{array}{l}\text { T0: } 7 \\
\text { T1: } 9\end{array}$ & $\begin{array}{c}\text { Anxiety-Related } \\
\text { Behaviors } \\
\text { Questionnaire }\end{array}$ & $\begin{array}{c}\text { Correlations } \\
\text { between } \mathrm{Mz} \\
\text { and } \mathrm{Dz} \\
\text { Structural Equation } \\
\text { Models } \\
\text { Correlations } \\
\text { between factors at } \\
\text { ages } 7 \text { and } 9\end{array}$ & $\begin{array}{c}\text { Phenotypical } \\
\text { correlation of } \\
0.54(0.54-0.56) \\
\text { between ages } 7 \\
\text { and } 9 \\
\text { Correlation } \\
\text { differences } \\
\text { between } \mathrm{MZ} \text { and } \\
\mathrm{DZ} \text { in the two ages }\end{array}$ & $17(77 \%)$ \\
\hline $\begin{array}{l}\text { Van Hulle et al., } \\
201247\end{array}$ & $\begin{array}{l}\text { USA/Twins } \\
\text { (medical record } \\
\text { services) }\end{array}$ & $\begin{array}{l}175 \mathrm{P} \mathrm{Mz} \\
150 \mathrm{P} \mathrm{Dz} \\
160 \mathrm{P} \mathrm{Dz} 0\end{array}$ & $\begin{array}{l}7.7 \\
(0.7)\end{array}$ & $\begin{array}{l}\text { Diagnostic } \\
\text { Interview Schedule } \\
\text { for Children }\end{array}$ & $\begin{array}{l}\text { Correlations } \\
\text { between } \mathrm{Mz} \\
\text { and } \mathrm{Dz}\end{array}$ & $\begin{array}{l}\text { Correlation } \\
\text { differences }\end{array}$ & $17(77 \%)$ \\
\hline $\begin{array}{l}\text { Waszczuk et al., } \\
201420\end{array}$ & $\begin{array}{l}\text { UK/Twins (medical } \\
\text { record services) }\end{array}$ & 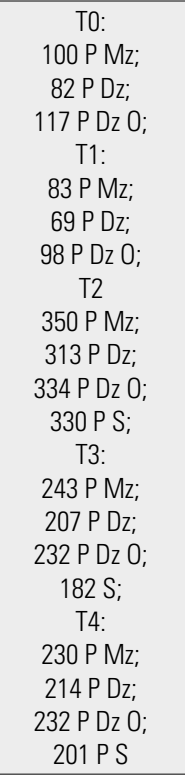 & $\begin{array}{c}\text { T0: } \\
8 \text { years and } 6 \\
\text { months } \\
\text { T1: } \\
10 \text { years and } 1 \\
\text { month } \\
\text { T2: } \\
15 \text { years } \\
\text { T3: } \\
17 \text { years } \\
\text { T4: } \\
20 \text { years }\end{array}$ & $\begin{array}{l}\text { SCARED for } \\
\text { children; } \\
\text { SCAS for } \\
\text { adolescents; } \\
\text { Revised } \\
\text { Symptoms of } \\
\text { Anxiety Scale for } \\
\text { adults }\end{array}$ & $\begin{array}{l}\text { Structural Equation } \\
\text { Models }\end{array}$ & $\begin{array}{l}\text { Heritability } \\
\text { estimates }\end{array}$ & $19(86 \%)$ \\
\hline
\end{tabular}

P: Pairs; I: Single Individuals; Mz: monozygotic twins; Dz: dizygotic twins; S: siblings; M: Male; F: Female; 0: Opposite Sex; SAD: Social Anxiety Disorder Case; GAD: Generalized Anxiety Disorder Case; PD: Panic Disorder Case; AD: Anxiety Disorder Case; MDD: Major Depression Disorder Case; APD: Avoidant Personality Disorder Case; HC: Health Control; OR: Odds ratio.

The analysis of correlational differences between monozygotic and dizygotic twins and heritability models that best explain the genetic and environmental contributions to SAD, in addition to estimates of each factor, are core elements for the assessment of heritability ${ }^{19}$. Therefore, we extracted the data from studies describing these variables, detailed in Table 2 . Among these articles, the model that best fit the sample in most studies $(66.7 \%)$ was the one that considers only additive genetic factors (A) and non-shared environmental factors (E). However, a significant share of the articles (28.6\%) proposed that shared environmental factors $(\mathrm{C})$ were an important component of the best explicative model. The study by Waszczuk et al. ${ }^{20}$, that assessed pairs of twins as they aged, was the only one that suggested two models as most adequate because of the sample characteristics. Specifically, the authors considered the ACE model to be the most adequate for children, whereas the AE model was regarded as the most adequate for adults. There was great variability in the estimated heritability rates across studies, with heritability measured according to additive genetic factors ranging between $13 \%$ and $76 \%$. 
Table 2. Correlation between Monozygotic and Dizygotic twins, model that best fits the sample, additive genetic effects, shared environmental effects and non-shared environmental effects of each study included

\begin{tabular}{|c|c|c|c|c|c|c|}
\hline Reference & $\mathrm{RMz}(\mathrm{Cl})$ & $\mathrm{RDz}(\mathrm{Cl})$ & Model & $\mathrm{A}(\mathrm{Cl})$ & $\mathrm{C}(\mathrm{Cl})$ & $E(C I)$ \\
\hline $\begin{array}{l}\text { Czajkowski et al., } \\
201122\end{array}$ & $0.56(0.32-0.73)$ & $0.14(0.23-0.48)$ & $\mathrm{AE}$ & 0.55 & - & 0.45 \\
\hline Eley et al., $2003^{23}$ & $\begin{array}{c}\text { Males }=0.57 \\
\text { Females }=0.56\end{array}$ & $\begin{array}{c}\text { Males }=0.02 \\
\text { Females }=0.13\end{array}$ & $\mathrm{AE}$ & $\begin{array}{c}\text { Males } \\
0.76(0.71-0.79) \\
\text { Females } \\
0.66(0.59-0.71)\end{array}$ & - & $\begin{array}{c}\text { Males } \\
0.24(0.21-0.29) \\
\text { Females } \\
0.34(0.29-0.41)\end{array}$ \\
\hline Eley et al., $2008^{24}$ & $0.27(-0.04-0.54)$ & $0.14(-.07-0.35)$ & ACE & $0.14(0.00-0.45)$ & $0.10(0.00-0.30)$ & $0.76(0.52-0.93)$ \\
\hline Hallett et al., 200914 & $\begin{array}{c}\mathrm{Mz7}=0.7(0.68-0.72) \\
\mathrm{Mz} 9=0.77(0.75-0.79)\end{array}$ & $\begin{array}{l}\mathrm{Dz7}=0.31(0.28-0.35) \\
\mathrm{Dz} 9=0.48(0.45-0.51)\end{array}$ & ACE & $\begin{array}{c}7 \text { years } \\
0.61(0.57-0.63) \\
9 \text { years } \\
0.56(0.48-0.63) \\
\end{array}$ & $\begin{array}{c}7 \text { Years } \\
0.07 \text { (0.05-0.1) } \\
9 \text { Years } \\
0.2(0.13-0.26) \\
\end{array}$ & $\begin{array}{c}7 \text { years } \\
0.32(0.3-0.33) \\
9 \text { years } \\
0.25(0.23-0.27) \\
\end{array}$ \\
\hline Hallett et al., 201225 & $\begin{array}{l}\mathrm{MzM}=0.7(0.67-0.72) \\
\mathrm{MzF}=0.69(0.66-0.73)\end{array}$ & $\begin{array}{l}\mathrm{DzM}=0.30(0.29-0.33) \\
\mathrm{DzF}=0.34(0.32-0.36)\end{array}$ & ACE & $\begin{array}{c}\text { Males } \\
0.6(0.54-0.66) \\
\text { Females } \\
0.59(0.53-0.65) \\
\end{array}$ & $\begin{array}{c}\text { Males } \\
0.09(0.04-0.14) \\
\text { Females } \\
0.10(0.03-0.17) \\
\end{array}$ & $\begin{array}{c}\text { Males } \\
0.31(0.27-0.35) \\
\text { Females } \\
0.31(0.27-0.35) \\
\end{array}$ \\
\hline Hettema et al., $2005^{10}$ & _ & _- & ACE & 0.1 & 0.11 & 0.79 \\
\hline Hettema et al., 200126 & _- & _- & ACE & 0.13 & 0.09 & 0.78 \\
\hline Isomura et al., $2015^{29}$ & _- & _ & _ & 0.56 & _- & _ \\
\hline Kendler et al., $2001^{30}$ & _ & _- & $\mathrm{AE}$ & $0.2(0-0.41)$ & _- & $0.8(0.59-1)$ \\
\hline Kendler et al., 200831 & $\begin{aligned} \text { MzF 13-14 } & =0.51 \\
\text { MzF 16-17 } & =0.52 \\
M z F \text { 19-20 } & =0.44 \\
\text { MzM 13-14 } & =0.45 \\
\text { MzM 16-17 } & =0.41 \\
\text { MzM 19-20 } & =0.17\end{aligned}$ & $\begin{aligned} \text { DzF 13-14 } & =0.17 \\
\text { DzF 16-17 } & =0.14 \\
\text { DzF 19-20 } & =0.08 \\
\text { DzM 13-14 } & =0.31 \\
\text { DzM 16-17 } & =0.14 \\
\text { DzM 19-20 } & =0.49\end{aligned}$ & $A E$ & $\begin{array}{l}13-14=0.49 \\
16-17=0.44 \\
19-20=0.34\end{array}$ & - & $\begin{array}{l}13-14=0.50 \\
16-17=0.55 \\
19-20=0.65\end{array}$ \\
\hline Lahey et al., 201133 & _- & - & $\mathrm{AE}$ & 0.45 & _- & 0.55 \\
\hline $\begin{array}{l}\text { López-Solà et al., } \\
201434\end{array}$ & $\begin{array}{c}\text { Mz Total = } 0.46 \text { (0.39- } \\
0.52) ; \mathrm{MzM}=0.38 \\
(0.25-0.49) ; \mathrm{MzF}= \\
0.49(0.40-0.56)\end{array}$ & $\begin{array}{c}\text { Dz TOTAL } \\
0.18(0.09-0.27) \\
\text { DzM } \\
0.07(0.11-0.25) \\
\text { DzF } \\
0.24(0.11-0.36) \\
\text { DzO } \\
0.16(0.01-0.32 \\
\end{array}$ & $A E$ & $\begin{array}{c}\text { Males } \\
0.34(0.23-0.45) \\
\text { Females } \\
0.47(0.39-0.55)\end{array}$ & - & $\begin{array}{c}\text { Males } \\
0.66(0.55-0.77) \\
\text { Females } \\
0.53(0.45-0.61)\end{array}$ \\
\hline Michelini et al., $2015^{37}$ & \begin{tabular}{|c|}
$\mathrm{MzM}=0.43(0.24-$ \\
$0.59)$ \\
$\mathrm{MzF}=0.34(0.17-0.48)$
\end{tabular} & $\begin{array}{c}\mathrm{DzM} / \mathrm{SM}=0.37(0.18- \\
0.54) ; \mathrm{DzF} / \mathrm{SF}=0.24 \\
(0.09-0.38) ; \mathrm{DzO} / \mathrm{SO}= \\
0.09(-0.04-0.21)\end{array}$ & $A E$ & $0.35(0.26-0.44)$ & - & $0.65(0.56-0.74)$ \\
\hline Mosing et al., 200939 & _ & _- & $\mathrm{AE}$ & $0.39(0.16-0.65)$ & _- & _ \\
\hline Nelson et al., $2000^{40}$ & - & - & $\mathrm{AE}$ & 0.28 (Common factor) & _ & 0.72 (Specific factor) \\
\hline Ogliari et al., 200641 & $\begin{array}{c}0.58 \text { (8-11 years) } \\
0.561 \text { (12-17 years) }\end{array}$ & $\begin{array}{c}0.26 \text { (8-11 years) } \\
0.303 \text { (8-11 years) }\end{array}$ & $A E$ & $0.56(0.46-0.66)$ & - & $0.44(0.34-0.54)$ \\
\hline Ogliari et al., $2010^{42}$ & $0.57(0.45-0.66)$ & $0.31(0.19-0.42)$ & $\mathrm{AE}$ & $0.56(0.46-0.65)$ & _ & $0.44(0.35-0.54)$ \\
\hline $\begin{array}{l}\text { Reichborn-Kjennerud } \\
\text { et al., } 200743\end{array}$ & $0.57(0.29-0.78)$ & $0.06(-0.41-0.50)$ & $\mathrm{AE}$ & 0.39 & - & 0.61 \\
\hline Skre et al., $2000^{44}$ & 0.53 & -0.02 & $\mathrm{AE}$ & 0.47 & _ & 0.53 \\
\hline Stein et al., $2002^{45}$ & $\begin{array}{l}\mathrm{MzM}=0.462 \\
\mathrm{MzF}=0.503\end{array}$ & $\begin{array}{l}\mathrm{DzM}=0.253 \\
\mathrm{DzF}=0.124 \\
\mathrm{DzO}=0.143\end{array}$ & $A E$ & $0.42(0.32-0.51)$ & - & $0.58(0.49-0.69)$ \\
\hline $\begin{array}{l}\text { Trzaskowski et al., } \\
2012^{46}\end{array}$ & $\begin{array}{c}7 \text { years }=0.70(0.68- \\
0.72) \\
9 \text { years }=0.77(0.75- \\
0.79)\end{array}$ & $\begin{array}{c}7 \text { years }=0.31(0.28- \\
0.35) \\
9 \text { years }=0.48(0.45- \\
0.51)\end{array}$ & ACE & $\begin{array}{c}7 \text { to } 9 \text { years } \\
0.66(0.59-0.66)\end{array}$ & $\begin{array}{c}7 \text { to } 9 \text { years } \\
0.55(0.35-0.75)\end{array}$ & $\begin{array}{c}7 \text { to } 9 \text { years } \\
0.42(0.37-0.42)\end{array}$ \\
\hline $\begin{array}{l}\text { Van Hulle et al., } \\
2012^{47}\end{array}$ & 0.39 & 0.09 & - & - & - & - \\
\hline
\end{tabular}




\begin{tabular}{|c|c|c|c|c|c|c|}
\hline Reference & $\mathrm{RMz}(\mathrm{Cl})$ & $\mathrm{RDz}$ (Cl) & Model & $\mathrm{A}(\mathrm{Cl})$ & $\mathrm{C}(\mathrm{Cl})$ & $E(C I)$ \\
\hline $\begin{array}{l}\text { Waszczuk et al., } \\
201420\end{array}$ & - & - & $\begin{array}{c}\text { ACE } \\
\text { (Children) } \\
\text { AE (Adults) }\end{array}$ & $\begin{array}{c}8 \text { years } \\
\text { Common factor } 0.12 \\
(0-0.24)+\text { Specific } \\
\text { factor } 0(0-0.07) \\
10 \text { years } \\
\text { Common factor }=0.38 \\
(0-0.53)+\text { Specific } \\
\text { factor }=0 \text { (0-0.42) } \\
\text { Adults } \\
\text { All variables factor }= \\
0.4(0.3-0.49) \\
\text { Fear model factor }= \\
0.07(0.01-0.12)\end{array}$ & $\begin{array}{c}8 \text { years } \\
\text { Common factor }=0.0 \\
(0-0.08)+\text { Specific } \\
\text { factor }=0(0-0.04) \\
10 \text { years } \\
\text { Common factor }=0.1 \\
(0-0.27)+\text { Specific } \\
\text { factor }=0(0-0.25)\end{array}$ & $\begin{array}{c}8 \text { years } \\
\text { Common factor }=0.28 \\
(0.17-0.46)+\text { Specific } \\
\text { factor }=0.59(0.49- \\
0.68) \\
10 \text { years } \\
\text { Common factor }=0.21 \\
(0.09-0.40)+\text { Specific } \\
\text { factor }=0.40(0.27- \\
0.54) \\
\text { Adults } \\
\text { Common factor }=0.26 \\
(0.18-0.34) \\
\text { Specific factor }=0.27 \\
(0.22-0.33)\end{array}$ \\
\hline
\end{tabular}

MzM: monozygotic males; MzF: monozygotic females; DzM: dizygotic males; DzF: dizygotic females; DzO: dizygotic different sex; SM: sibling males; SF: sibling females; SO: siblings different sex; RMz: correlations between monozygotic twins; RDz: correlations between dizygotic twins; Cl: confidence interval; A: additive genetic effects; C: shared environmental effects; E: non-shared environmental effects.

\section{Discussion}

The objective of this study was to systematically review articles assessing heritability to $\mathrm{SAD}$, with no limits regarding publication date and including different methodological designs. A total of 31 articles were included in the review, most of which involved pairs of twins as their sample. The studies were conducted mainly in Europe and used mostly self-reporting instruments to assess SAD symptoms. SAD heritability was estimated through correlation differences between twins and based structural equation models. We found that additive genetic factors and non-shared environmental factors formed the most adequate model to explain SAD heritability, with genetic transmission rates estimated between $13 \%$ and $76 \%$.

The vast majority of studies that recruited twins for their samples used birth and medical follow-up records, which allowed the enrollment of a large number of twins and provided a significant amount of information on the heritability of SAD. Conversely, only one study involving twins recruited participants by means of community advertisement. This study included fewer participants than the mean in twin studies, probably as a result of the difficulty of recruiting participants through this method.

Generally speaking, genetic studies for heritability estimates require large samples 48,49 in order to ensure the statistical power of their analyses. It is thus important to encourage the creation and maintenance of records about twins, especially in low- and middleincome countries. This fact becomes evident in the present review, as no articles from developing countries were included due to not using methodological designs compatible with the investigation of SAD heritability.

Clinical interviews are regarded as the gold standard for the diagnosis of SAD and are widely used both as validation parameters for instruments that assess SAD symptoms $\left(e . g . .^{50,51}\right)$ and as a criterion for the selection of participants in clinical trials (e.g.: $\left.{ }^{52,53}\right)$ and genetic investigations (e.g.: ${ }^{21,28}$ ). Nevertheless, in order to be effective as diagnostic instruments, clinical interviews must be performed within a short interval from enrollment in studies because of the longitudinal instability of psychiatric diagnoses. Some of the articles reviewed here (e.g.: ${ }^{29}$ ) mentioned the limitation of using previous medical records without later diagnostic confirmation, which affects the reliability of the data presented.

An important feature of clinical interviews is that their outcome is a dichotomous variable, that is, a positive or negative diagnosis. On the other hand, a number of initiatives have been made for the adoption of dimensional criteria in the assessment of mental disorders (54,55), aiming at greater adequacy of the diagnostic process. These measures tend to bring the clinical setting closer to basic research, providing a more global comprehension of psychopathology, especially in the case of SAD, since social inhibition is an innate aspect of humans and thus of little accuracy for the establishment of psychiatric diagnosis. Particularly in genetic research, such initiatives may allow a closer association between the factors that influence a disorder and the symptoms of this disorder ${ }^{56}$. In this direction, the use of instruments that assess symptoms within a disorder continuum and that offer dimensional criteria for disorder assessment is of great importance ${ }^{57}$.

Most of the studies included in this review used this type of instrument, enabling the assessment of different possibilities of symptom manifestations. However, in order to provide reliable data, the instruments must present minimally adequate psychometric qualities and be compatible with the research interests. An important issue in some of the articles reviewed here was the use of instruments that were not subject to validation studies (e.g.: $\left.{ }^{31}\right)$ or versions derived from other instruments without the conduction of new psychometric evaluations ${ }^{14}$.

The concept of heritability is broad and generally refers to the proportion of the variance that can be explained by genetic factors ${ }^{58}$. As a result of the broadness of the concept, different methods can be employed to observe its occurrence in a given condition. The diversity in the methods used by the studies reviewed may have been a consequence of this variability. A high amount of variance explained by the fact of being related to a patient diagnosed with SAD in factorial analyses, the increased risk of being diagnosed with the disorder when a relative has received the same diagnosis (and the decrease in risk as genetic distance increases), and differences in the incidence of SAD between relatives with anxiety disorders suggest that heritability is an important variable in SAD 17,18,29. Nonetheless, although evidence points to the participation of genetic factors in SAD, it is not yet possible to determine the degree to which these conditions contribute for the effective determination of SAD diagnosis.

According to this issue, the enrollment of pairs of twins in the studies fosters relevant progress in the study of SAD heritability. Some of the studies included in this review, for instance, estimated heritability based on correlational differences between monozygotic and dizygotic twins. This method provides a clearer assessment of the contribution of genetic factors, to the extent that it allows a certain control over common external environmental variables. However, even with this outcome, it is not yet possible to determine the precise influence of genetics in the manifestation of SAD.

The use of structural equation models associated with the inclusion of samples of twins is, therefore, an advance in this direction. With this design, it is possible to develop models that explain better how genetic and environmental factors interact within a given sample to cause SAD, including also estimates of how much of the variance can be explained by each factor. However, although 
the articles reviewed did use these methods, we still observed a large amplitude in the variance explained by genetic factors in this review, which hinders precise estimates of these factors. Likewise, the diversity of results does not allow for a common interpretation of gender-related differences, nor of aspects such as the variability between age ranges regarding the establishment of diagnoses. We thus suggest the performance of studies involving carefully selected samples and instruments with adequate psychometric qualities.

One limitation of our review is the exclusion of studies that may have described results related to the heritability of SAD as secondary outcomes. Another possible limitation is the large variability in the instruments, methods, and outcomes in the studies reviewed, which hinders the homogenization in the presentation of results. This same limitation, however, ends up as an important part of this study as it describes the several ways that can used to assess heritability in SAD. Likewise, the conclusions of this review might contribute for the development of further research in this specific field.

In general terms, we conclude that heritability has been investigated in SAD through different methodological approaches, providing important evidence for a better comprehension of the factors that participate in the development of the condition. Future studies involving homogeneous samples and standardized instruments that allow a better diagnostic assessment of SAD would contribute for the estimation of more accurate heritability rates and for a better comprehension of the genetic factors associated with SAD.

\section{Acknowledgements}

ALM receives grant from CAPES-PROEX; RMS receives the support of the Generalitat de Catalunya: SGR2014/1114; JASC receives a CNPq Research Productivity Award (1B).

\section{References}

1. Kessler RC, Petukhova M, Sampson NA, Zaslavsky AM, Wittchen H-U. Twelve-month and lifetime prevalence and lifetime morbid risk of anxiety and mood disorders in the United States. Int J Methods Psychiatr Res. 2012;21(3):169-84.

2. Kessler RC, Chiu WT, Demler O, Merikangas KR, Walters EE. Prevalence, severity, and comorbidity of 12-month DSM-IV disorders in the National Comorbidity Survey Replication. Arch Gen Psychiatry. 2005;62(6):617-27.

3. Simon NM, Otto MW, Korbly NB, Peters PM, Nicolaou DC, Pollack MH. Quality of life in social anxiety disorder compared with panic disorder and the general population. Psychiatr Serv. 2002;53(6):714-8.

4. Katzelnick DJ, Greist JH. Social anxiety disorder: an unrecognized problem in primary care. J Clin Psychiatry. 2001;62 Suppl 1:11-6.

5. Ollendick TH, Hirshfeld-Becker DR. The developmental psychopathology of social anxiety disorder. Biol Psychiatry. 2002;51(1):44-58.

6. Furmark T, Appel L, Henningsson S, Åhs F, Faria V, Linnman C, et al. A link between serotonin-related gene polymorphisms, amygdala activity, and placebo-induced relief from social anxiety. J Neurosci. 2008;28(49):13066-74.

7. Biederman J, Hirshfeld-Becker DR, Rosenbaum JF, Hérot C, Friedman D, Snidman N, et al. Further evidence of association between behavioral inhibition and social anxiety in children. Am J Psychiatry. 2001;158(10):1673-9.

8. Caster JB, Inderbitzen HM, Hope D. Relationship between youth and parent perceptions of family environment and social anxiety. J Anxiety Disord. 1999;13(3):237-51.

9. Johnson HD, Lavoie JC, Mahoney M. Interparental conflict and family cohesion: predictors of loneliness, social anxiety, and social avoidance in late adolescence. J Adolesc Res. 2001;16(3):304-18.

10. Hettema JM, Neale MC, Kendler KS. A review and meta-analysis of the genetic epidemiology of anxiety disorders. Am J Psychiatry. 2001;158(10):1568-78.

11. Scaini S, Belotti R, Ogliari A. Genetic and environmental contributions to social anxiety across different ages: a meta-analytic approach to twin data. J Anxiety Disord. 2014;28(7):650-6.
12. Von Elm E, Altman DG, Egger M, Pocock SJ, Gøtzsche PC, Vandenbroucke JP; STROBE Initiative. The Strengthening the Reporting of Observational Studies in Epidemiology (STROBE) statement: guidelines for reporting observational studies. PLoS Med. 2007:4(10):e296.

13. Connor KM, Davidson JR, Churchill LE, Sherwood A, Foa E, Weisler RH. Psychometric properties of the Social Phobia Inventory (SPIN). New self-rating scale. Br J Psychiatry. 2000;176:379-86.

14. Hallett V, Ronald A, Rijsdijk F, Eley TC. Phenotypic and genetic differentiation of anxiety-related behaviors in middle childhood. Depress Anxiety. 2009;26(4):316-24.

15. First MB, Spitzer RL, Gibbon M, Williams JBW. Structured Clinical Interview for DSM-IV Axis I Disorders - Clinician Version (SCID-CV). Washington (DC): American Psychiatric Press; 1997.

16. World Health Organization. Composite International Diagnostic Interview. Geneva: World Health Organization; 1990.

17. Stein MB, Chartier MJ, Lizak MV, Jang KL. Familial aggregation of anxietyrelated quantitative traits in generalized social phobia: clues to understanding "disorder" heritability? Am J Med Genet. 2001;105(1):79-83.

18. Li X, Sundquist J, Sundquist K. Sibling risk of anxiety disorders based on hospitalizations in Sweden. Psychiatry Clin Neurosci. 2011;65(3):233-8.

19. Boomsma D, Busjahn A, Peltonen L. Classical twin studies and beyond. Nat Rev Genet. 2002;3(11):872-82.

20. Waszczuk MA, Zavos HM, Gregory AM, Eley TC. The phenotypic and genetic structure of depression and anxiety disorder symptoms in childhood, adolescence, and young adulthood. JAMA Psychiatry. 2014;71(8):905-16.

21. Coelho HF, Cooper PJ, Murray L. A family study of co-morbidity between generalized social phobia and generalized anxiety disorder in a non-clinic sample. J Affect Disord. 2007;100(1-3):103-13.

22. Czajkowski N, Kendler KS, Tambs K, Røysamb E, Reichborn-Kjennerud $\mathrm{T}$. The structure of genetic and environmental risk factors for phobias in women. Psychol Med. 2011;41(9):1987-95.

23. Eley TC, Bolton D, O'Connor TG, Perrin S, Smith P, Plomin R. A twin study of anxiety-related behaviours in pre-school children. J Child Psychol Psychiatry. 2003;44(7):945-60.

24. Eley TC, Rijsdijk FV, Perrin S, O'Connor TG, Bolton D. A multivariate genetic analysis of specific phobia, separation anxiety and social phobia in early childhood. J Abnorm Child Psychol. 2008;36(6):839-48.

25. Hallett V, Ronald A, Rijsdijk F, Happé F. Disentangling the associations between autistic-like and internalizing traits: a community based twin study. J Abnorm Child Psychol. 2012;40(5):815-27.

26. Hettema JM, Prescott CA, Myers JM, Neale MC, Kendler KS. The structure of genetic and environmental risk factors for anxiety disorders in men and women. Arch Gen Psychiatry. 2005;62(2):182-9.

27. Hettema JM, Neale MC, Myers JM, Prescott CA, Kendler KS. A population-based twin study of the relationship between neuroticism and internalizing disorders. Am J Psychiatry. 2006;163(5):857-64.

28. Hudson JI, Mangweth B, Pope HG Jr, De Col C, Hausmann A, Gutweniger S, et al. Family study of affective spectrum disorder. Arch Gen Psychiatry. 2003;60(2):170-7.

29. Isomura $K$, Boman $M$, Rück $C$, Serlachius E, Larsson $H$, Lichtenstein $\mathrm{P}$, et al. Population-based, multi-generational family clustering study of social anxiety disorder and avoidant personality disorder. Psychol Med. 2015;45(8):1581-9.

30. Kendler KS, Myers J, Prescott CA, Neale MC. The genetic epidemiology of irrational fears and phobias in men. Arch Gen Psychiatry. 2001;58(3):257-65.

31. Kendler KS, Gardner CO, Annas P, Lichtenstein P. The development of fears from early adolesence to young adulthood: a multivariate study. Psychol Med. 2008;38(12):1759-69.

32. Knappe S, Beesdo K, Fehm L, Lieb R, Wittchen HU. Associations of familial risk factors with social fears and social phobia: evidence for the continuum hypothesis in social anxiety disorder? J Neural Transm (Vienna). 2009;116(6):639-48.

33. Lahey BB, Van Hulle CA, Singh AL, Waldman ID, Rathouz PJ. Higherorder genetic and environmental structure of prevalent forms of child and adolescent psychopathology. Arch Gen Psychiatry. 2011;68(2):181-9.

34. López-Solà C, Fontenelle LF, Alonso $\mathrm{P}$, Cuadras D, Foley DL, Pantelis $\mathrm{C}$, et al. Prevalence and heritability of obsessive-compulsive spectrum and anxiety disorder symptoms: A survey of the Australian Twin Registry. Am J Med Genet B Neuropsychiatr Genet. 2014;165B(4):314-25. 
35. Low NC, Cui L, Merikangas KR. Specificity of familial transmission of anxiety and comorbid disorders. J Psychiatr Res. 2008;42(7):596-604.

36. Low NC, Cui L, Merikangas KR. Community versus clinic sampling: effect on the familial aggregation of anxiety disorders. Biol Psychiatry. 2008;63(9):884-90.

37. Michelini G, Eley TC, Gregory AM, McAdams TA. Aetiological overlap between anxiety and attention deficit hyperactivity symptom dimensions in adolescence. J Child Psychol Psychiatry. 2015;56(4):423-31.

38. Middeldorp CM, Birley AJ, Cath DC, Gillespie NA, Willemsen G, Statham DJ, et al. Familial clustering of major depression and anxiety disorders in Australian and Dutch twins and siblings. Twin Res Hum Genet. 2005;8(6):609-15.

39. Mosing MA, Gordon SD, Medland SE, Statham DJ, Nelson EC, Heath $\mathrm{AC}$, et al. Genetic and environmental influences on the co-morbidity between depression, panic disorder, agoraphobia, and social phobia: a twin study. Depress Anxiety. 2009;26(11):1004-11.

40. Nelson EC, Grant JD, Bucholz KK, Glowinski A, Madden PAF, Reich $\mathrm{W}$, et al. Social phobia in a population-based female adolescent twin sample: co-morbidity and associated suicide-related symptoms. Psychol Med. 2000;30(4):797-804.

41. Ogliari A, Citterio A, Zanoni A, Fagnani C, Patriarca V, Cirrincione $\mathrm{R}$, et al. Genetic and environmental influences on anxiety dimensions in Italian twins evaluated with the SCARED questionnaire. J Anxiety Disord. 2006;20(6):760-77.

42. Ogliari A, Spatola CA, Pesenti-Gritti P, Medda E, Penna L, Stazi MA, et al. The role of genes and environment in shaping co-occurrence of DSM-IV defined anxiety dimensions among Italian twins aged 8-17. J Anxiety Disord. 2010;24(4):433-9.

43. Reichborn-Kjennerud T, Czajkowski N, Torgersen S, Neale MC, Ørstavik $\mathrm{RE}$, Tambs K, et al. The relationship between avoidant personality disorder and social phobia: a population-based twin study. Am J Psychiatry. 2007;164(11):1722-8.

44. Skre I, Onstad S, Torgersen S, Philos DR, Lygren S, Kringlen E. The heritability of common phobic fear: a twin study of a clinical sample. J Anxiety Disord. 2000;14(6):549-62.

45. Stein MB, Jang KL, Livesley WJ. Heritability of social anxiety-related concerns and personality characteristics: a twin study. J Nerv Ment Dis. 2002;190(4):219-24.
46. Trzaskowski M, Zavos HM, Haworth CM, Plomin R, Eley TC. Stable genetic influence on anxiety-related behaviours across middle childhood. J Abnorm Child Psychol. 2012;40(1):85-94.

47. Van Hulle CA, Schmidt NL, Goldsmith HH. Is sensory over-responsivity distinguishable from childhood behavior problems? A phenotypic and genetic analysis. J Child Psychol Psychiatry. 2012;53(1):64-72.

48. Hong EP, Park JW. Sample size and statistical power calculation in genetic association studies. Genomics Inform. 2012;10(2):117-22.

49. Stram DO. Design of large-scale genetic association studies, sample size, and power. In: Stram DO. Design, analysis, and interpretation of genome-wide association scans. New York: Springer; 2014.

50. de Lima Osório F, Crippa JA, Loureiro SR. A study of the discriminative validity of a screening tool (MINI-SPIN) for social anxiety disorder applied to Brazilian university students. Eur Psychiatry. 2007;22(4):23943.

51. Dalrymple K, Martinez J, Tepe E, Young D, Chelminski I, Morgan T, et al. A clinically useful social anxiety disorder outcome scale. Compr Psychiatry. 2013;54(7):758-65.

52. Anderson PL, Price M, Edwards SM, Obasaju MA, Schmertz SK, Zimand E, et al. Virtual reality exposure therapy for social anxiety disorder: a randomized controlled trial. J Consult Clin Psychol. 2013;81(5):751-60.

53. Hedman E, Andersson G, Ljótsson B, Andersson E, Rück C, Mörtberg E, et al. Internet-based cognitive behavior therapy vs. cognitive behavioral group therapy for social anxiety disorder: a randomized controlled noninferiority trial. PLoS One. 2011;6(3):e18001.

54. Craske MG. The R-DoC initiative: science and practice. Depress Anxiety. 2012;29(4):253-6.

55. Regier DA, Narrow WE, Kuhl EA, Kupfer DJ. The conceptual development of DSM-V. Am J Psychiatry. 2009;166(6):645-50.

56. Simmons JM, Quinn KJ. The NIMH Research Domain Criteria (RDoC) Project: implications for genetics research. Mamm Genome. 2014;25(12):23-31.

57. Lebeau RT, Glenn DE, Hanover LN, Beesdo-Baum K, Wittchen HU, Craske MG. A dimensional approach to measuring anxiety for DSM-5. Int J Methods Psychiatr Res. 2012;21(4):258-72.

58. Visscher P, Hill WG, Wray NR. Heritability in the genomics era concepts and misconceptions. Nat Rev Genet. 2008;9(4):255-66. 